\title{
Bounds on Maximum Throughput for Digital Communications with Finite-Precision and Amplitude Constraints
}

\author{
MICHAEL L. HONIG, MEMBER, IEEE, KENNETH STEIGLITZ, FELLOW, IEEE, \\ B. GOPINATH, MEMBER, IEEE, AND STEPHEN P. BOYD, MEMBER, IEEE
}

\begin{abstract}
The problem of finding the maximum achievable data rate over a linear time-invariant channel is considered under constraints different from those typically assumed. The limiting factor is taken to be the accuracy with which the receiver can measure the channel output. More precisely, we consider the following problem. Given a channel with known impulse response $h(t)$, a transmitter with an output amplitude constraint, and a receiver that can distinguish between two signals only if they are separated in amplitude at some time $t_{0}$ by at least some small positive constant $d$, what is the maximum number of messages, $N_{\max }$, that can be transmitted in a given time interval $[0, T]$ ? Lower bounds on $N_{\max }$ can be easily computed by constructing a particular set of inputs to the channel. Our main result is an upper bound on $N_{\max }$ for arbitrary $h(t)$. The upper bound depends on the spread of $h(t)$, which is the maximum range of values the channel output may take at some time $t_{0}>0$ given that the output takes on a particular value $\alpha$ at time $t=0$. For a particular $h(t)$, computing the spread in discrete-time is equivalent to solving a linear program with bounded variables and one equality constraint. Solutions to linear programs in this class can be obtained very fast using, for example, a linear-time algorithm due to Witzgall. Numerical results are shown for different impulse responses, including two simulated telephone subscriber loop impulse responses. Assuming that the receiver resolution $d$ is small, the upper bound is typically two to four times the lower bound for the cases examined.
\end{abstract}

\section{INTRODUCTION}

$\mathrm{I}$

$\mathrm{N}$ THE MOST elementary view of a communication channel, we choose from among a set of $N$ possible signals to transmit, and must then distinguish which was transmitted by measurements at the receiver over a finite length of time $T$. If we limit the resources that can be used at the transmitter, characterize the distortion caused by the transmission channel, and limit the accuracy with which the received signal can be measured to $\pm d / 2$, there is some maximum value to $N$ for a given signaling

Manuscript received July 7, 1988; revised April 7, 1989. This work was presented in part at the 1988 International Conference on Acoustics, Speech, and Signal Processing, New York, NY, April 11-14, 1988. K. Steiglitz was supported in part by the NSF Grant MIP-8912100, and ARO-Durham Contract DAAL03-89-K-0074.

M. L. Honig is with Bell Communications Research, 445 South St. Room MRE 2L-343, Morristown, NJ 07960-1910.

K. Steiglitz is with the Department of Computer Science, Princeton, NJ 08544 .

B. Gopinath is with the Department of Electrical Engineering, Rutgers University, Piscataway, NJ 08855.

S. P. Boyd is with the Information Systems Laboratory, Department of

Electrical Engineering. Stanford University, Stanford, CA 94305.

IEEE Log Number 8933981. time $T$, say $N_{\max }(T, d)$. The maximum channel throughput (MCT), can then be defined by

$$
\operatorname{MCT}(d)=\lim _{T \rightarrow \infty} \frac{\log N_{\max }(T, d)}{T} \mathrm{~b} / \mathrm{s} .
$$

This formulation for bounding communication rate was anticipated as early as 1928 by Hartley [1] (also see [2]).

This definition of channel throughput is motivated by the study of high-speed transmission over single twistedwire pairs. Transmission errors in this case are caused by effects that are not easily modeled by random noise, such as precision of analog-to-digital conversion, circuit nonlinearities, timing inaccuracies, and residual intersymbol interference. Thus, the MCT is an entirely deterministic notion, in contrast with the Shannon capacity, which is based on a probabilistic model of the channel.

We will consider here the problem of estimating this MCT in situations where the limiting resource at the transmitter is the dynamic range of the electronics, which leads naturally to an amplitude constraint on the transmitter waveforms, and where the channel can be accurately modeled as linear and time-invariant. Furthermore, we will assume that the uncertainty in the amplitude of the received signal is determined by a single parameter $d$, the receiver discrimination. More precisely, we assume that two received signals can be distinguished when and only when they differ in amplitude by at least $d$ at some point in the time interval $[0, T]$. If $u_{1}, \cdots, u_{N}$ are inputs to the channel defined on $[0, T]$ and $y_{1}, \cdots, y_{N}$ are the corresponding outputs, then

$$
\begin{aligned}
& N_{\max }(T, d) \\
& =\max _{u_{1}, \cdots, u_{N}}\left\{N\left|\min _{\substack{i \neq j \\
1 \leq i, j \leq N}} \sup _{0 \leq t \leq T}\right| y_{i}(t)-y_{j}(t) \mid \geq d\right\}
\end{aligned}
$$

where it is assumed that $|u(t)| \leq 1$ for all $t$. Put another way, the MCT is a measure of achievable transmission rate with a limit on the $L_{x}$ norm of the input, and an $L_{x}$ criterion for discrimination at the receiver. The corresponding notion using the $L_{2}$ norm at both transmitter and receiver is called $\epsilon$-capacity [3]. 
It is shown in [4] that $N_{\text {max }}(T, d)$ is easily evaluated for the case $h(t)=A e^{-\alpha t}$. It is also shown that there exists a maximizing set of inputs $u_{1}, \cdots, u_{N}$ such that $\left|u_{j}(t)\right|=1$ for all $t$. Here we give upper and lower bounds on $N_{\max }(T, d)$ for any $T>0, d>0$, and impulse response $h(t)$.

\section{A Lower Bound on Throughput}

The output of the channel at time $t$ is $y(t)=h * u(t)$, where $u(t)$ is the input, $h(t)$ is the channel impulse response, and the asterisk denotes convolution. The input that maximizes the output at time $T$, subject to the constraint $|u(s)| \leq 1,0 \leq s \leq T$, is clearly

$$
u^{*}(t)=\operatorname{sign} h(T-t), \quad 0 \leq t \leq T,
$$

in which case the output at time $T$ is

$$
C(T) \equiv \int_{0}^{T}|h(t)| d t
$$

The minimum time it takes to distinguish $N$ channel outputs is defined as

$$
T_{\min }(N, d)=\min _{u_{1}, \cdots, u_{N}}\left\{T\left|\min _{i \neq j} \sup _{0 \leq t \leq T}\right| y_{i}(t)-y_{j}(t) \mid \geq d\right\}
$$

where it is assumed that $d<2 \int_{0}^{\infty}|h(t)| d t$. For the case $N=2$ a set of inputs that achieves $T_{0} \equiv T_{\min }(2, d)$ is clearly $u_{1}(t)=-u_{2}(t)=u^{*}(t)$, where $T_{0}$ satisfies

$$
C\left(T_{0}\right)=\frac{d}{2} \text {. }
$$

We can define a mapping of $M$ source bits $\left(N=2^{M}\right.$ messages) to a channel input $u_{i}(t), 0 \leq t \leq M T$, as follows:

$$
u_{i}(t)=\sum_{k=1}^{M} b_{i, k} u_{0}[t-(k-1) T]
$$

where $b_{i, k}$ is either 1 or -1 corresponding to the $k$ th bit of message $i, 1 \leq i \leq 2^{M}$, and $u_{0}(t)=u^{*}(t), 0 \leq t \leq T$, $u_{0}(t)=0, t>T$. If the period $T=T_{0}$ as given by (2.4), then, using linearity, it is easily verified that

$$
\max _{0 \leq t \leq M T}\left|h * u_{i}(t)-h * u_{j}(t)\right| \geq d
$$

for any $i \neq j$. In this case the time it takes to distinguish $2^{M}$ messages is exactly $M T_{0}$.

Consider now applying the preceding inputs to a channel with impulse response $h(t)=t$, and restrict the number of inputs to be $N=4$. Fig. 1 shows the outputs corresponding to four inputs given by (2.5), where the $b_{i, k}$ are the four possible two bit sequences. Since $h(t)>0$, $t>0$, the inputs are $u_{1}(t)=-u_{2}(t)=1,0 \leq t \leq 2 T$, and

$$
u_{3}(t)=-u_{4}(t)=\left\{\begin{aligned}
1, & 0 \leq t \leq T \\
-1, & T<t \leq 2 T
\end{aligned}\right.
$$

Because of the overshoot that occurs when the input switches from 1 to -1 , or vice versa, if $T=T_{0}$, then the maximum distance between any two distinct outputs over the interval $[0,2 T+\delta]$, where $\delta$ is a fixed sampling delay,

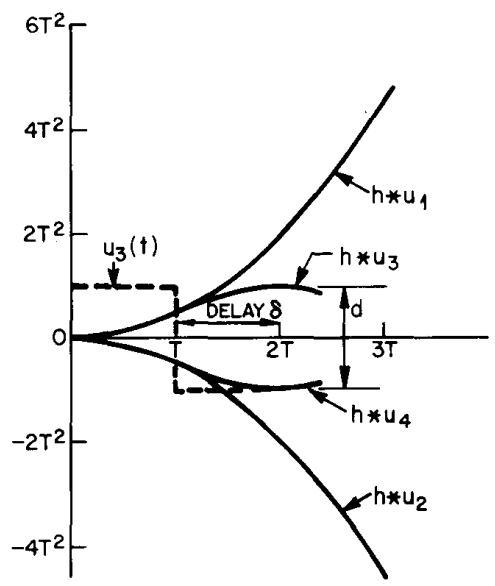

Fig. 1. Outputs $t * u_{i}(t)$ where $u_{1}(t)=1, u_{2}(t)=-1, u_{3}(t)=-u_{4}(t)$, and $u_{3}(t)$ switches from 1 to -1 .

is greater than $d$. The period $T$ can therefore be decreased to $T^{\prime}<T_{0}$ (thereby increasing the rate) so that $\max _{0 \leq t \leq 2 T^{\prime}+\delta}\left|h * u_{i}(t)-h * u_{j}(t)\right|=d, \quad i \neq j$. It is assumed in Fig. 1 that $T=T^{\prime}$.

Given that the inputs have the form (2.5), the output of the channel can be sampled at times $n T+\delta, n=1,2, \cdots$, and a simple threshold detector can be used to detect each bit sequentially. In particular, if at time $t_{i j}=n T+\delta$, $y_{i}\left(t_{i j}\right)-y_{j}\left(t_{i j}\right)=d$, where $y_{i}(t)=h * u_{i}(t)$, then the threshold level is set to $\gamma_{i j}=\left[y_{i}\left(t_{i j}\right)+y_{j}\left(t_{i j}\right)\right] / 2$. If the received output $r\left(t_{i j}\right)>\gamma_{i j}$, then the receiver excludes $u_{j}$ as a possible transmitted signal, and if $r\left(t_{i j}\right)<\gamma_{i j}$, then $u_{i}$ is excluded. The sampling phase $\delta$ should be chosen so that the distance between outputs at sampling times is maximized. Fig. 1 indicates that for the minimum $T$ there may be a fixed delay $\delta$ between when a bit is transmitted, and when it is detected. A more revealing illustration of this point is given in Fig. 2, which shows an impulse response consisting of two pulses separated by a delay. The optimal delay $\delta$ that maximizes the distance $d$ is shown in Fig. 2.

Theorem 1: Given any $h(t)$ and discrimination $d>0$, then

$$
\operatorname{MCT}(d) \geq \frac{1}{T}
$$

where $T$ satisfies

$$
\begin{aligned}
\max _{\delta}\left(\int_{\delta}^{\delta+T}|h(t)| d t-\int_{0}^{\delta}|h(t)| d t\right) \\
=\max _{\delta}[C(\delta+T)-2 C(\delta)]=\frac{d}{2}
\end{aligned}
$$

Proof: Consider the set of inputs having the form (2.5), where

$$
u_{0}(t)= \begin{cases}\operatorname{sgn} h(T+\delta-t), & 0 \leq t<T \\ 0, & t>T\end{cases}
$$

and $\delta$ and $T$ are chosen according to (2.8). Assume that for the distinct inputs $u_{i}$ and $u_{j}, b_{i, k_{0}}=-b_{j, k_{0}}$ for some 
$k_{0} \geq 1$ and that $b_{i, k}=b_{j, k}$ for $k<k_{0}$. Then

$$
\begin{aligned}
\left|y_{i}\left(k_{0} T+\delta\right)-y_{j}\left(k_{0} T+\delta\right)\right| & \left|\int_{0}^{k_{0} T+\delta} h\left(k_{0} T+\delta-s\right)\left(u_{i}(s)-u_{j}(s)\right) d s\right| \\
= & \mid \int_{\left(k_{0}-1\right) T}^{k_{0} T} h\left(k_{0} T+\delta-s\right)\left(b_{i, k_{0}}-b_{j, k_{0}}\right) \\
& \cdot u_{0}\left[s-\left(k_{0}-1\right) T\right] d s \\
& +\int_{0}^{\left(k_{01}-1\right) T} h\left(k_{0} T+\delta-s\right) \\
& \cdot \sum_{k<k_{0}}\left(b_{i, k}-b_{j, k}\right) u_{0}[s-(k-1) T] d s \\
& +\int_{k_{0}, T}^{k_{0} T+\delta} h\left(k_{0} T+\delta-s\right) \\
& \cdot \sum_{k>k_{0}}\left(b_{i, k}-b_{j, k}\right) u_{0}[s-(k-1) T] d s \mid \\
= & \mid\left(b_{i, k_{0}}-b_{j, k_{0}}\right) \int_{\left(k_{0}-1\right) T}^{k_{0} T} h\left(k_{0} T+\delta-s\right) \\
& \cdot \operatorname{sgn} h\left(k_{0} T+\delta-s\right) d s \\
& +\int_{0}^{\delta} h\left(\delta-t^{\prime}\right)\left[\sum_{k>k_{0}}\left(b_{i, k}-b_{j, k}\right)\right. \\
& \left.\cdot u_{0}\left(t^{\prime}+\left(k_{0}-k\right) T\right)\right] d t^{\prime} \mid \\
& 2 \int_{\delta}^{\delta+T}\left|h\left(t^{\prime}\right)\right| d t^{\prime}-2 \int_{0}^{\delta}\left|h\left(t^{\prime}\right)\right| d t^{\prime}=d, \quad(2.10
\end{aligned}
$$

since $T$ and $\delta$ are chosen so that (2.8) is satisfied. Consequently, with the set of inputs previously defined, $k_{0}$ bits can be communicated in time $k_{0} T+\delta$, so that

$$
\begin{aligned}
\operatorname{MCT}(d) & =\lim _{k_{11} \rightarrow \infty} \frac{\log N_{\max }\left(k_{0} T+\delta, d\right)}{k_{0} T+\delta} \\
& \geq \lim _{k_{11} \rightarrow \infty} \frac{k_{0}}{k_{0} T+\delta}=\frac{1}{T} . \quad \square
\end{aligned}
$$

If $h(t) \geq 0$ for all $t,(2.8)$ says that if the input is initially 1 , and subsequently switches to -1 at time $T$, then the maximum value of the output is $d / 2$. If $h(t)=A e^{-\alpha t}$ where $A$ and $\alpha$ are positive constants, then the $\delta$ that maximizes the left-hand side of (2.8) is zero for any $T$. Consequently, the preceding lower bound for the MCT is $1 / T$ where $C(T)=d / 2$. It is shown in [4] that this is exactly the MCT, so that the method for constructing inputs (2.8), (2.9) maximizes the data rate in this case. Of course, it is apparent that this method gives a data rate equal to the MCT whenever the optimal inputs have the form (2.5).

\section{An UPPER BOUnd on ThroughPut}

One way of upper bounding the number of distinguishable signals, $N_{\max }$, is to consider the following question: Given that we have control over the (bounded) input of the channel, and that the output of the channel is constrained to take on a given value $\alpha$ at a given time $t$, how far apart can we spread the output at some future time $T$ ? Let $h$ denote the channel impulse response, so that the output at time $t$ is $y(t)=h * u(t)$, where $u$ is the input and the asterisk denotes convolution. Then the largest and smallest outputs at time $T$ are

$$
\begin{aligned}
\sigma^{+}(T-t, \alpha)= & \sup _{\substack{u(s) \\
-x<s \leq T}}\{y(T)|y(t)=\alpha,| u(s) \mid \leq 1\} \\
\sigma^{-}(T-t, \alpha)= & \inf _{\substack{u(s) \\
-\infty<s \leq T}}\{y(T)|y(t)=\alpha,| u(s) \mid \leq 1\}
\end{aligned}
$$

and the spread function is defined to be, for $\tau \geq 0$,

$$
\sigma(\tau, \alpha)=\sigma^{+}(\tau, \alpha)-\sigma^{-}(\tau, \alpha)
$$

The spread function is not necessarily a monotonically increasing function of $\tau$.

Before proceeding to the precise statement of results, we first give an overview and discussion of what follows. For a large set of impulse responses $\boldsymbol{H}$, which will subsequently be defined, we prove the following upper bound on the MCT. For any impulse response in $\boldsymbol{H}$ let $\tilde{\tau}$ be the first time at which the spread $\sigma(\tilde{\tau}, 0)=d$. Then $\operatorname{MCT}(d) \leq 1 / \tilde{\tau}$ in bits $/$ s. The reason for choosing $\alpha=0$ is that this value maximizes the spread for any fixed $\tau$ (see Theorem 3). An alternative statement of this upper bound is that for any $h(\cdot) \in \boldsymbol{H}$, if the data rate is $R$, then the maximum separation between pairs of outputs can be no greater than $\sigma(1 / R, 0)$.

It is easily verified that this upper bound is always greater than or equal to the lower bound given by Theorem 1. In particular, if it is assumed that the inputs have the form given by $(2.5)$, then the symbol period $T$ must be greater than $\tilde{\tau}$ to guarantee a separation of $d$ between any two distinct outputs. Otherwise, if $T<\tilde{\tau}$, then by the definition of $\tilde{\tau}$, two channel inputs corresponding to messages which differ in only bit cannot "spread apart" by $d$ in one symbol period $T$.

Although our upper bound is not very tight for many impulse responses in the set $\boldsymbol{H}$ (see the numerical examples in Section VII), it gives the exact MCT for the case of the exponential impulse response $h(t)=A e^{-\alpha t}, \alpha \geq 0$ (which is in the set $\boldsymbol{H}$ ). In this case the state of the channel at time $t$ can be taken to be the output $y(t)$. Consequently, given $y(t)$, the output at time $T$, where $T>t$, is independent of the input $u(s)$ for $s<t$. The function $\sigma^{+}\left(\sigma^{-}\right)$is therefore determined by selecting the input $u(s)$ for $t<s<T$ to maximize (minimize) $y(T)$, i.e., $u(s)=1(-1)$. In this case we therefore have that $\sigma^{ \pm}(\tau, 0)$ $= \pm C(\tau)$, where $C(\tau)$ is defined by (2.2). Therefore $\sigma(\tau, 0)=2 C(\tau)$, and the upper bound agrees with the lower bound given by Theorem 1 (see the discussion 

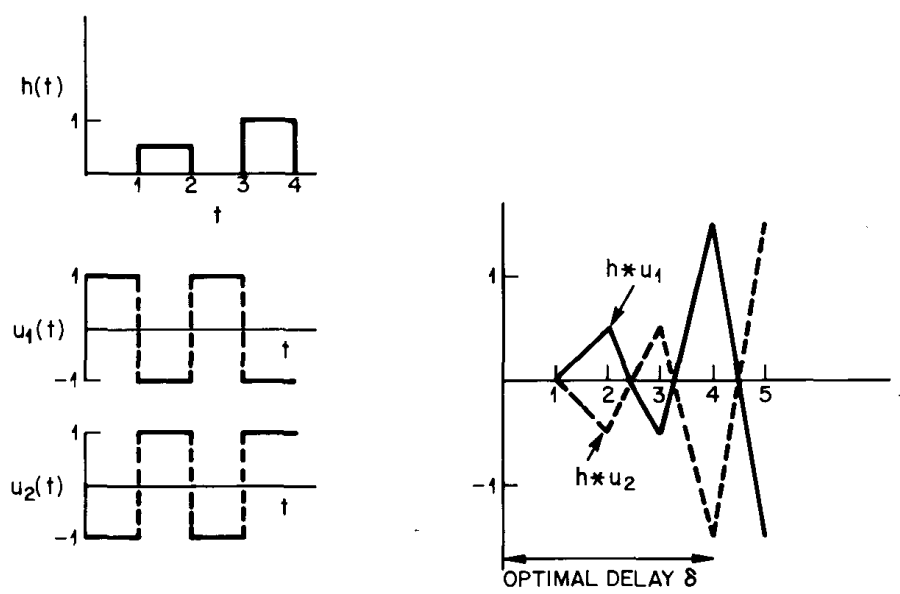

Fig. 2. Impulse response for which optimal sampling delay $\delta>0$.

following (2.11)). In general, of course, $y(T)$ depends not only on $y(t)$, but also on $u(s)$ for $s<t$, so that $\sigma(\tau, 0)$ will be strictly greater than $C(\tau)$, which implies that the preceding upper bound on MCT will be strictly greater than the lower bound.

The proof of the preceding upper bound assumes that the spread function has certain monotonicity properties, which are defined in the Appendix. The set $\boldsymbol{H}$ is the set of impulse responses for which the associated spread functions satisfy these properties. Conditions that an impulse response must satisfy to be in $\boldsymbol{H}$ are derived in the Appendix (see Theorems 7 and 8), and are stated in (3.8), (3.9). Although we have not been able to prove that the preceding upper bound holds for any $h(t)$, neither have we found a counterexample. That is, we have not found an $h(t) \notin \boldsymbol{H}$ and a set of inputs such that the resulting lower bound on MCT is greater than $1 / \tilde{\tau}$. Whether or not such an impulse response exists remains an open question.

An upper bound on MCT that holds for arbitrary bounded and piecewise continuous $h(t)$ can be obtained by modifying the spread function in such a way that the monotonicity properties referred to in the preceding paragraph are always valid. This new modified spread function, $\bar{S}(\tau, \alpha)$, has the property that $\bar{S}(\tau, \alpha) \geq \sigma(\tau, \alpha)$ for any $\tau$ and $\alpha$ (see (3.3), (3.4)). For any impulse response $h(t)$ let $\tau^{*}$ be the first time at which $\bar{S}\left(\tau^{*}, 0\right)=d$ (see (3.6)). Then it will be shown that $\operatorname{MCT}(d) \leq 1 / \tau^{*}$. If $h(t) \in \boldsymbol{H}$, then $\tau^{*}=\tilde{\tau}$; however, in general, $\tau^{*}<\tilde{\tau}$ for $h(t) \notin \boldsymbol{H}$.

The rest of the paper is organized as follows. Our main result is Theorem 2, which, given any bounded and piecewise continuous impulse response, states a lower bound on the minimum time it takes to distinguish $N$ channel outputs for fixed receiver discrimination $d$. This directly implies the preceding upper bound on MCT, and an upper bound on the maximum separation between channel outputs for a fixed number of inputs and time interval $[0, T]$ (see $(3.12),(3.13))$. Computation of the spread func- tion, which determines the upper bound on MCT for $h \in \boldsymbol{H}$, is then considered in Sections IV-VI. In general, computation of the spread for arbitrary $h(t)$ is difficult (see [8]). However, in the discrete-time case it is shown in Section IV that the calculation of spread is equivalent to solving a linear program with bounded variables and one equality constraint. The derivation of a fast algorithm for solving linear programs in this class is presented in Sections V and VI. Upper and lower bounds on MCT for some specific sampled impulse responses are subsequently presented in Section VII. Two of the examples correspond to existing subscriber loop twisted-wire pairs.

\section{A. Statement of Results}

To compute an upper bound on the MCT for arbitrary $h(t)$, we define the following functions

$$
\begin{aligned}
& S^{+}(t, \alpha)=\sup _{\substack{0 \leq s \leq t \\
-\|h\|_{1} \leq \alpha^{\prime} \leq \alpha}} \sigma^{+}\left(s, \alpha^{\prime}\right) \\
& S^{-}(t, \alpha)=\inf _{\substack{0 \leq s \leq t \\
\alpha \leq \alpha^{\prime} \leq\|h\|_{1}}} \sigma^{-}\left(s, \alpha^{\prime}\right)
\end{aligned}
$$

and

$$
\begin{aligned}
& \bar{S}^{+}(t, \alpha)=\alpha+\sup _{\alpha \leq \alpha^{\prime} \leq\|h\|_{t}}\left(S^{+}\left(t, \alpha^{\prime}\right)-\alpha^{\prime}\right) \\
& \bar{S}^{-}(t, \alpha)=\alpha-\inf _{-\|h\|_{1} \leq \alpha^{\prime} \leq \alpha}\left(\alpha^{\prime}-S^{-}\left(t, \alpha^{\prime}\right)\right)
\end{aligned}
$$

where $\|h\|_{1}=\int_{0}^{\infty}|h(s)| d s$. Also, $\quad \bar{S}(t, \alpha)=\bar{S}^{+}(t, \alpha)-$ $\bar{S}^{-}(t, \alpha)$, and $S(t, \alpha)$ is defined similarly. Note that $\sigma(\tau, \alpha) \leq \bar{S}(\tau, \alpha)$ for any $\tau$ and $\alpha$.

The proof of the next theorem is given in the Appendix. Throughout the rest of the paper it will be assumed that $h(\cdot)$ is bounded and piecewise continuous.

Theorem 2: For any impulse response $h$, discrimination $d>0$, and positive integer $N$,

$$
T_{\min }(N, d) \geq n \tau^{*}
$$

where $T_{\min }(N, d)$ is defined by $(2.3), 2^{n-1}<N \leq 2^{n}$, and 


$$
\tau^{*}=\inf \left\{\tau \mid \sup _{\alpha} \bar{S}(\tau, \alpha)=d\right\}
$$

It will be shown (see Theorem 7) that $\sup _{\alpha} \bar{S}(\tau, \alpha)=$ $\vec{S}(\tau, 0)$.

Let

$$
\tilde{\tau}=\inf \left\{\tau \mid \sup _{\alpha} \sigma(\tau, \alpha)=d\right\}
$$

Note that $\tau^{*} \leq \tilde{\tau}$ for any $h$. It is shown in the Appendix that if the impulse response $h(t)$ satisfies

$$
\int_{\boldsymbol{B}(t)}|h(-s)| d s \leq \int_{\bar{B}(t)}|h(-s)| d s
$$

where

$$
\begin{aligned}
& \boldsymbol{B}(t)=\left\{s \mid \frac{h(t-s)}{h(-s)}>1, \quad s \leq 0\right\}, \\
& \overline{\boldsymbol{B}}(t)=\left\{s \mid \frac{h(t-s)}{h(-s)}<1, \quad s \leq 0\right\},
\end{aligned}
$$

at time $t=\tau^{*}$, then $\tau^{*}=\tilde{\tau}$. For the impulse response functions considered in this paper, this condition is true for all $t$ so that the simpler upper bound $\mathrm{MCT} \leq 1 / \tilde{\tau}$ applies. Note, in particular, that (3.8) is true for any positive and nonincreasing impulse response. An impulse response for which (3.8) is false for some values of $t$ is $h(s)=s, 0<s \leq t_{0}, h(s)=0$ elsewhere.

Theorem 2 implies that

$$
N_{\max }(T, d) \leq 2^{\left[T / \tau^{*} \mid\right.},
$$

for all positive $T$ and $d$, so that

$$
\operatorname{MCT}(d) \leq \frac{1}{\tau^{*}} .
$$

Defining the max-min distance among $N$ outputs in a given time interval $[0, T]$ as

$$
d_{\max }(N, T)=\max _{u_{1}, \cdots, u_{N}} \min _{i \neq j} \sup _{0 \leq t \leq T}\left|y_{i}(t)-y_{j}(t)\right|
$$

where $1 \leq i, j \leq N,\left|u_{i}(t)\right| \leq 1$ for all $t>0$, and $y_{i}(0)=0$ for each $i$, then Theorem 2 and the proof of Theorem 1 imply that

$$
d_{\max }\left(2^{n}, n \tau\right) \leq \sup _{t<\tau} \bar{S}(t, 0)
$$

and

$$
d_{\max }\left(2^{n}, n \tau+\delta^{*}\right) \geq \sup _{\delta} 2(C(\tau+\delta)-2 C(\delta))
$$

for all $\tau>0$, and positive integers $n$, where $\delta^{*}$ is the value of $\delta$ that achieves the supremum on the right.

Solving the optimization problems embodied in (3.1) and (3.2) enables us to upper bound the maximum throughput of the channel. Before proceeding to the computation of the spread function, we first prove some elementary facts about $\sigma^{+}$and $\sigma^{-}$.
Theorem 3: For any fixed $\tau, \sigma^{+}(\tau, \alpha)$ is a concave function of $\alpha$. Also,

1) $\sigma^{+}(\tau, \alpha)=-\sigma^{-}(\tau,-\alpha)$

2) $\sup _{\alpha} \sigma(\tau, \alpha)=\sigma^{+}(\tau, 0)-\sigma^{-}(\tau, 0)$.

Proof: Without loss of generality we can assume that $t=0$ in (3.1). Let $u_{1}(s)$ and $u_{2}(s)$ be inputs such that $\sigma^{+}\left(T, \alpha_{1}\right)=h * u_{1}(T)$ and $\sigma^{+}\left(T, \alpha_{2}\right)=h * u_{2}(T)$, so that $h * u_{1}(0)=\alpha_{1}$ and $h * u_{2}(0)=\alpha_{2}$. Consequently,

$$
\begin{aligned}
h *\left[\theta u_{1}+(1-\theta) u_{2}\right](0) & =\theta h * u_{1}(0)+(1-\theta) h * u_{2}(0) \\
& =\theta \alpha_{1}+(1-\theta) \alpha_{2}
\end{aligned}
$$

where $0 \leq \theta \leq 1$, and

$$
\begin{aligned}
\theta \sigma^{+} & \left(T, \alpha_{1}\right)+(1-\theta) \sigma^{+}\left(T, \alpha_{2}\right) \\
& =\theta h * u_{1}(T)+(1-\theta) h * u_{2}(T) \\
& =h *\left[\theta u_{1}+(1-\theta) u_{2}\right](T) \\
& \leq \sigma^{+}\left[T, \theta \alpha_{1}+(1-\theta) \alpha_{2}\right],
\end{aligned}
$$

which proves that $\sigma^{+}$is a concave function of $\alpha$. Property 1 ) is obvious from the definition of $\sigma^{+}$and $\sigma^{-}$. Property 1) implies that $\sigma^{-}(\tau, \alpha)$ is convex in $\alpha$, so that $\sigma^{+}(\tau, \alpha)-$ $\sigma^{-}(\tau, \alpha)$ is concave and is an even function of $\alpha$. Consequently, $\sup _{\alpha}\left(\sigma^{+}-\sigma^{-}\right)$occurs at $\alpha=0$.

\section{Discrete-Time Formulation and Linear Program}

To calculate the spread function in practical situations, we consider the discrete-time case, and formulate the optimization problems as linear programs. The channel impulse response $h(t)$ and input $u(t)$ are therefore vectors, and $t, T$, and $s$ are integer-valued. Continuous-time computation of spread is considered in [8]. To make the problem have a finite number of variables, we pick $T$ large enough so that the impulse response is negligibly small for $s>T$, and consider the signal only in the range $0 \leq s \leq T$. The output of the channel at time $T$ is

$$
y(T)=\sum_{s=0}^{T} h(T-s) u(s) .
$$

The calculation of $\sigma^{+}$then becomes

$$
\max \sum_{s=0}^{T} h(T-s) u(s)
$$

subject to the constraints

$$
\begin{aligned}
\sum_{s=0}^{t} h(t-s) u(s) & =\alpha \\
|u(s)| \leq 1, \quad s=0, \cdots, T . &
\end{aligned}
$$

This is a linear program with $T+1$ variables constrained to lie in the unit cube, with one additional equality constraint. To simplify the notation, we rewrite (4.2)-(4.4) 
as

$$
\begin{gathered}
\max _{s} g^{\prime} x \\
f^{\prime} x=\alpha \\
|x| \leq 1
\end{gathered}
$$

where $g$ is the vector of impulse response coefficients and $f$ is a shifted version of $g$.

\section{Characterization of Solutions}

It is interesting to note that the dual program to (4.5) is the unconstrained $l_{1}$ approximation problem

$$
\min _{\lambda}\left\{\|g-\lambda f\|_{1}+\lambda \alpha\right\}
$$

where $\|x\|_{1}=\sum_{i}\left|x_{i}\right|$. This problem was considered by Laplace, among others [5], who gave an $O(n \log n)$ algorithm for finding the optimal $\lambda$. Witzgall [6] shows that the linear program (4.5) can be solved directly by using a "weighted median" algorithm. Depending on the method for finding the weighted median, this leads to $O(n \log n)$ and $O(n)$ algorithms. We will next present a direct derivation of a somewhat different $O(n \log n)$ algorithm, which will be fast enough for our application.

We begin with some observations that will allow us to simplify the discussion without loss of generality. Note first that if $\alpha$ is negative, we can multiply the single constraint equation by -1 , so we will assume $\alpha \geq 0$. Note next that if any $f_{i}=0$ we can take $x_{i}=\operatorname{sgn}\left(g_{i}\right)$ and effectively eliminate $x_{i}$ from the problem. Also, if $f_{i}<0$, we can replace $x_{i}, f_{i}$, and $g_{i}$ by $-x_{i},-f_{i}$, and $-g_{i}$, respectively, again without changing the problem. Therefore, we will assume that $f_{i}>0$ for all $i$. Finally, it will be convenient to order the ratios $g / f$ in decreasing order, $g_{1} / f_{1} \geq g_{2} / f_{2} \geq \cdots \geq g_{n} / f_{n}$.

The problem described by (4.5) is that of maximizing a linear function on the set resulting from the intersection of a hyperplane and the unit cube. Intuitively, there should always be a solution that lies on a bounding edge of the hypercube. This can be stated formally as follows.

Theorem 4: For given vectors $g$ and $f$ there is an integer $k$ and optimal solution $x$ to the linear program (4.5) with the property that $\left|x_{i}\right|=1$ for all $i \neq k$.

Proof: Suppose in an optimal solution there are two indexes $j, k, j<k$ such that $\left|x_{j}\right| \neq 1$ and $\left|x_{k}\right| \neq 1$. By optimality, $x_{j}$ and $x_{k}$ solve the problem

$$
\begin{gathered}
\max g_{j} x_{j}+g_{k} x_{k} \\
\text { subject to } f_{j} x_{j}+f_{k} x_{k}=c
\end{gathered}
$$

where $c$ is a constant.

Consider what happens if we replace $x_{j}$ by $x_{j}+\delta_{j}$ and $x_{k}$ by $x_{k}+\delta_{k}$. In order to keep $f^{\prime} x=c$ we must have

$$
f_{j} \delta_{j}+f_{k} \delta_{k}=0
$$

Using that fact, the change in the cost of the solution is

$$
\delta_{j} f_{j}\left(g_{j} / f_{j}-g_{k} / f_{k}\right) \text {. }
$$

If $g_{j} / f_{j}=g_{k} / f_{k}$, the cost is unaffected, so we can increase $\delta_{j}$ until either $\left|x_{j}\right|$ or $\left|x_{k}\right|=1$. Otherwise, we can increase the cost by choosing $\delta_{j}$ positive, which is a contradiction.

From now on, we will always use the symbol $k$ to represent an index with this property

$$
\left|x_{i}\right|=1, \quad i \neq k \text {. }
$$

We can now characterize the solution more precisely.

Theorem 5: There is always an optimal solution with the property (5.6) for which

$$
x_{j}= \begin{cases}+1, & j<k \\ -1, & j>k .\end{cases}
$$

Proof: Choose an optimal solution satisfying (5.6) and suppose first that $\left|x_{k}\right|<1$ and the ratios $g_{i} / f_{i}, \quad i=$ $1,2,3, \cdots, n$ are distinct.

If $j<k$ it follows from (5.5) that increasing $x_{j}$ while respecting (5.4) increases the cost, so it must be that $x_{j}=+1$, by optimality. Similarly, if $j>k$ we would be able to increase the cost by decreasing $x_{j}$, so $x_{j}=-1$.

When $x_{k}=+1$, the same argument shows that all $x_{j}$ to the left of $x_{k}$ are +1 , but not necessarily that the $x_{j}$ to the right are -1 . In this case, we can satisfy the conditions of the theorem by changing $k$ to the position of the rightmost +1 in the sequence of $x$ values. Symmetrically, choose the leftmost -1 when $x_{k}=-1$.

Finally we can deal with the case when the $g_{j} / f_{j}$ are not distinct by a perturbation argument. In that case, replace the vector $g$ by $g+\epsilon^{(t)}$, where $\epsilon^{(t)}$ is a sequence of vectors that converges to zero, in such a way as to keep the ratios $g_{j} / f_{j}$ distinct. From the corresponding sequence of optimal solutions, choose an infinite subsequence with the same value of $k=K$. Every member of this subsequence must be the same optimal solution given by the conditions of the theorem and

$$
x_{K}=\left(\alpha-\sum_{j \neq K} f_{j} x_{j}\right) / f_{K} .
$$

\section{AN $O(n \log n)$ Algorithm}

Theorem 5 states that one way to solve the linear program (4.5) is to find the set of indices $k$ such that

$$
\sum_{i=1}^{k-1} f_{i}-\sum_{i=k+1}^{n} f_{i}+f_{k} x_{k}=\alpha, \quad \text { where }\left|x_{k}\right| \leq 1 .
$$

Theorem 6: Given any real vector $f$ and positive constant $\alpha$, where $f_{i}>0$ and $\sum_{i=1}^{n} f_{i} \geq \alpha$, then there exists a unique $k$ such that either $(6.1)$ is true or

$$
\sum_{i=1}^{k} f_{i}-\sum_{i=k+1}^{n} f_{i}=\alpha \text {. }
$$

Proof: Let

$$
s_{j}=\alpha-\sum_{i=1}^{j} f_{i}+\sum_{i=j+1}^{n} f_{i} .
$$


Then $s_{j}+f_{j}=f_{j} x_{j}$ and

$$
s_{j}=s_{j-1}-2 f_{j} \text {. }
$$

$\left|x_{k}\right| \leq 1$ implies that $\left|s_{k}+f_{k}\right| \leq f_{k}$, or

$$
-2 f_{k} \leq s_{k} \leq 0 \text {. }
$$

If $s_{k}=0$ or $s_{k}=-2 f_{k}$, then (6.4) implies that (6.5) and (6.2) are satisfied for unique $k$. If $-2 f_{k}<s_{k}<0$, then (6.4) implies that $s_{j}<-2 f_{j}$ for $j>k$, and $s_{j}>0$ for $j<k$. Consequently, (6.5) is satisfied for a unique $k$.

It is now easy to see how to solve the problem: Starting with $k=1$ we have the following.

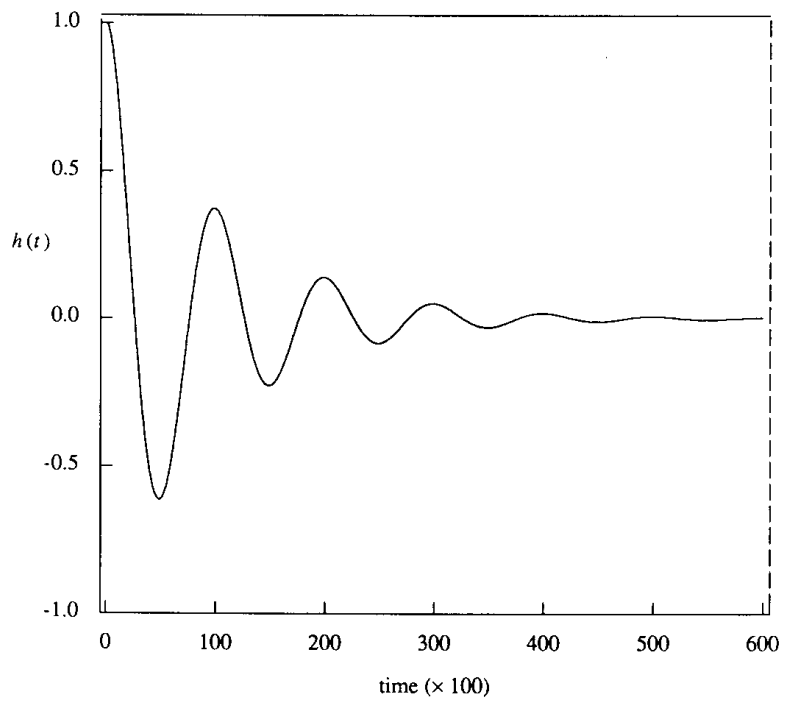

(a)
1) Choose $x_{j}$ according to (5.7) and calculate

$$
x_{k}=F_{k}=\left(\alpha-\sum_{j \neq k} f_{j} x_{j}\right) / f_{k} .
$$

2) If $\left|x_{k}\right| \leq 1$ stop. Otherwise, increment $k$.

If an optimal solution exists, we are assured of at least one such $x_{k}$ by Theorem 5. If the original problem is infeasible, no $x_{k}$ can be feasible.

The $F_{k}$ can be computed recursively using a constant number of operations for each value of $k$. Remembering the assumptions $\alpha \geq 0$ and $f_{i}>0$, the recursive relation

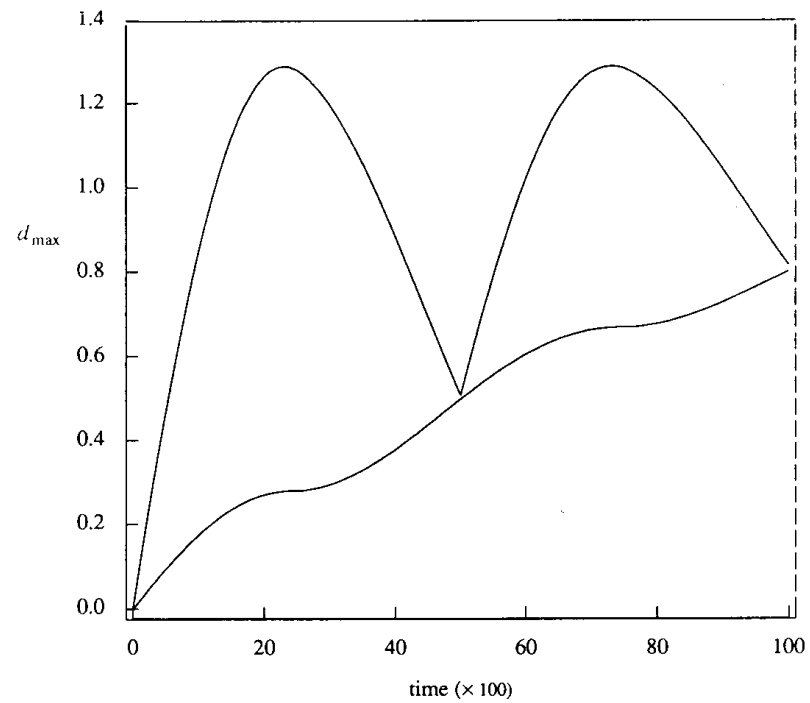

(b)

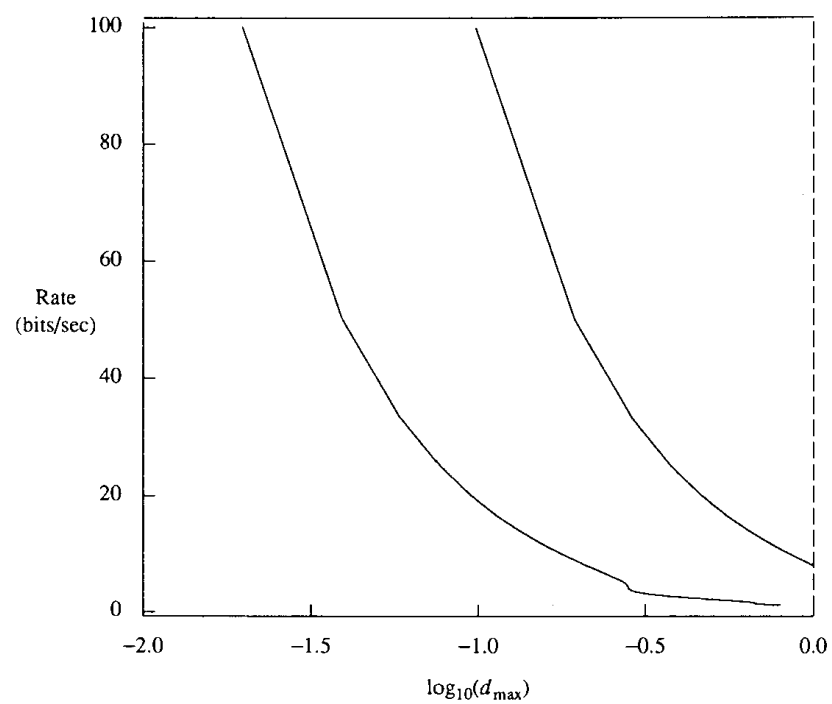

(c)

Fig. 3. (a) Oscillatory Impulse Response. (b) Upper and lower bounds on $d_{\max }$ for impulse response in (a). Top curve is $\sigma(t, 0)$ and bottom curve is lower bound (3.13b). (c) Upper and lower bounds on MCT for impulse response in (a). 
can be written

$$
\begin{gathered}
f_{1} F_{1}=\alpha+\sum_{i>1} f_{i} \\
f_{k+1} F_{k+1}=f_{k} F_{k}-f_{k+1}-f_{k} .
\end{gathered}
$$

In this way, after sorting the $g / f$ ratios, the solution can be found in an additional $O(n)$ arithmetic operations, and the entire solution in $O(n \log n)$ steps.

It has been pointed out by Witzgall [6] that the $k$ for which (6.1) or (6.2) is true can be found in $O(n)$ operations without sorting the $g / f$ ratios by using a median finding algorithm. Consequently, the solution to the linear program (4.5) can be found in linear time $(O(n))$. Never-

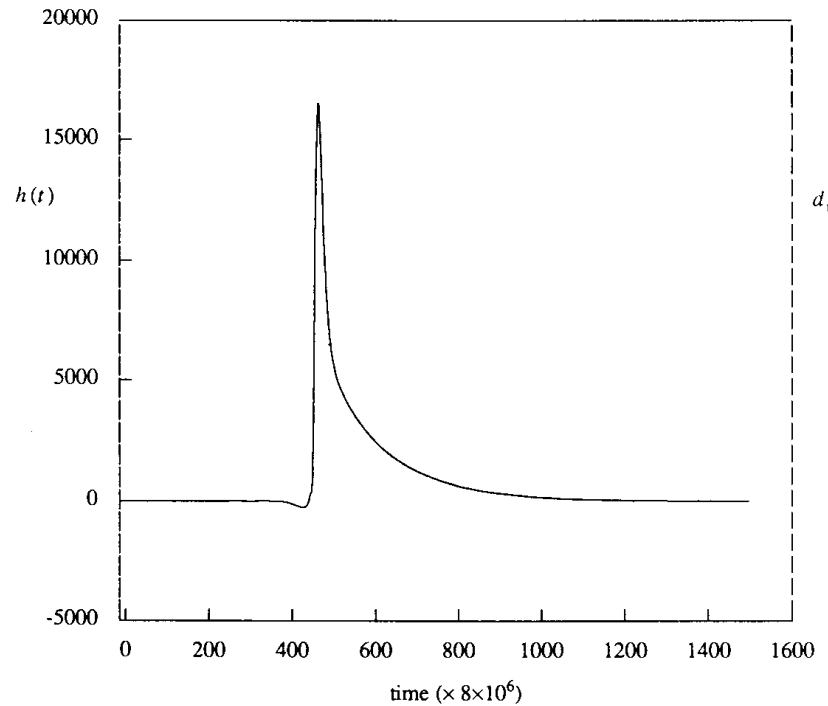

(a) theless, the $n \log n$ term in the time complexity of our algorithm is due only to the sorting operation, and therefore has a very small constant associated with it. In our case $n$ is never more than 1000 , so our algorithm is preferable in this application.

\section{Numerical Results}

According to (3.13) and Theorems 7 and 8, $\sup _{t^{\prime}<1} \sigma\left(t^{\prime}, 0\right)$ is an upper bound on the max-min distance $d_{\text {max }}\left(2^{n}, n t\right)$, defined by (3.12), provided that $(3.8)$ is true. An equivalent condition to $(3.8)$, which is easy to check for the discrete-time problem, is that $\lambda \leq 1$, where

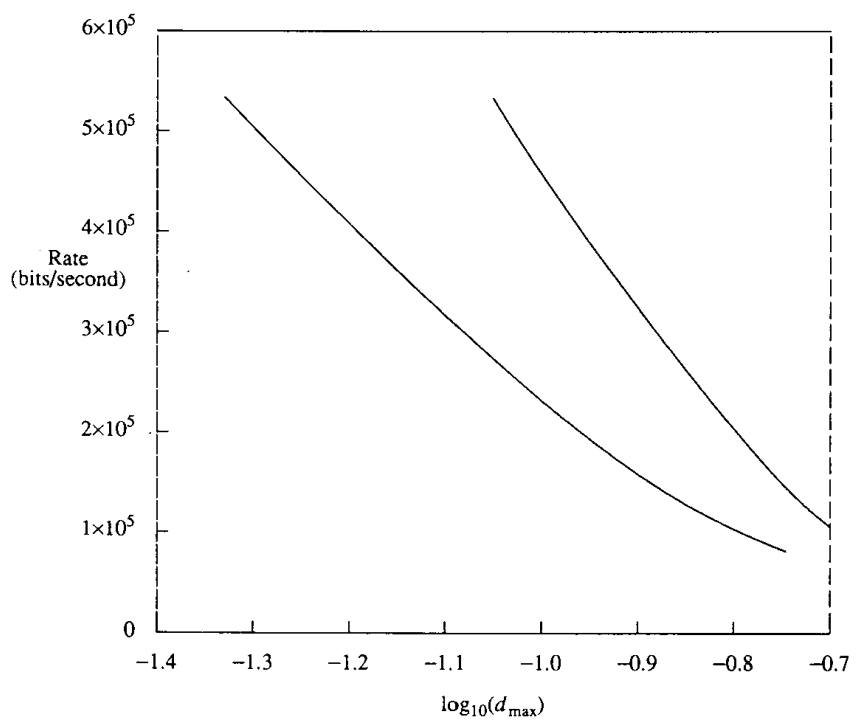

(c)

Fig. 4. (a) Subscriber loop impulse response. (b) Upper and lower bounds on $d_{\max }$ for impulse response in (a). (c) Upper and lower bounds on MCT for impulse response in (a). 
$\lambda$ is the solution to the $l_{1}$ approximation problem (5.1), where $\alpha=0$. This can easily be derived from the proof of Theorem 8 and the fact that (5.1) is the dual program to (4.5). In addition, it is easily shown [5] that $\lambda=g_{k} / f_{k}$, where $k$ is the index specified by (5.7). In each of the following three examples it was verified numerically that $\lambda \leq 1$ for each (discrete) $t$.

In the first example, we take the impulse response to be a sampled version of the artificially generated damped sinusoidal function

$$
h(t)=e^{-t} \cos 2 \pi t, \quad t \geq 0 .
$$

Fig. 3(a) shows the impulse response, and Fig. 3(b) shows

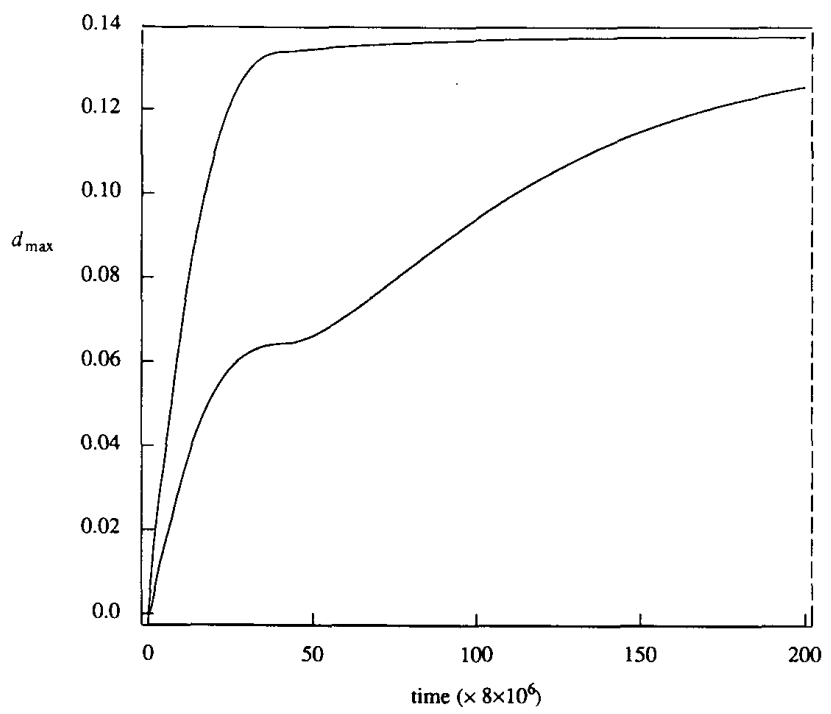

(a) the spread function

$$
\sigma(T-t, 0)=\sigma^{+}(T-t, 0)-\sigma^{-}(T-t, 0)
$$

where the signal is constrained to pass through the value $\alpha=0$ when $t=5$. (There are 100 samples per unit time.) Also shown in Fig. 3(b) is the lower bound on $d_{\max }$ given by $(3.13 b)$. It is interesting to note that the spread oscillates in synchronism with the impulse response, and actually comes down to touch the lower bound at periodic points. Of course, the MCT $(d)$ defined by (1.1) will nevertheless be a monotonically decreasing function of $d$. Upper and lower bounds on $\operatorname{MCT}(d)$ for this case are shown in Fig. 3(c).

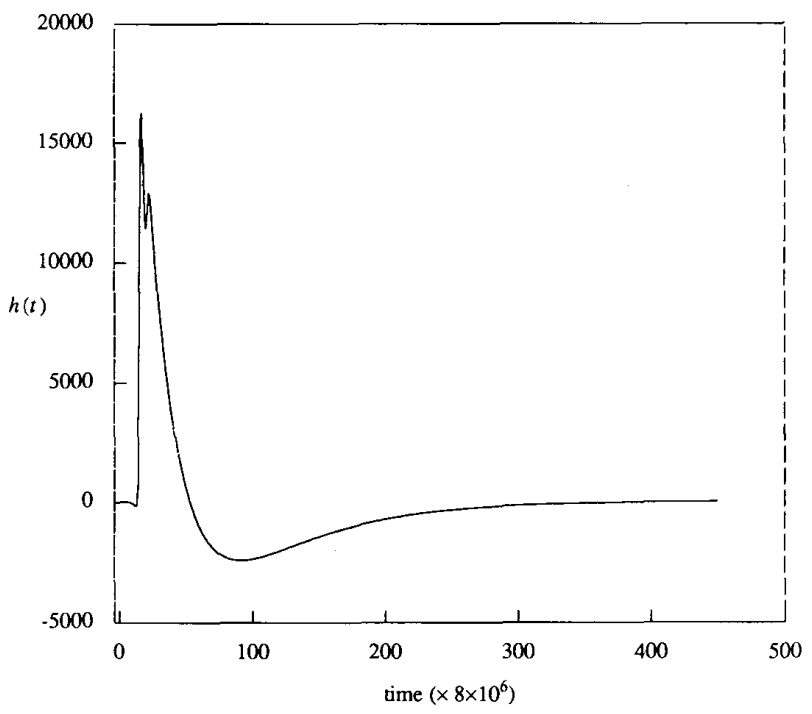

(b)

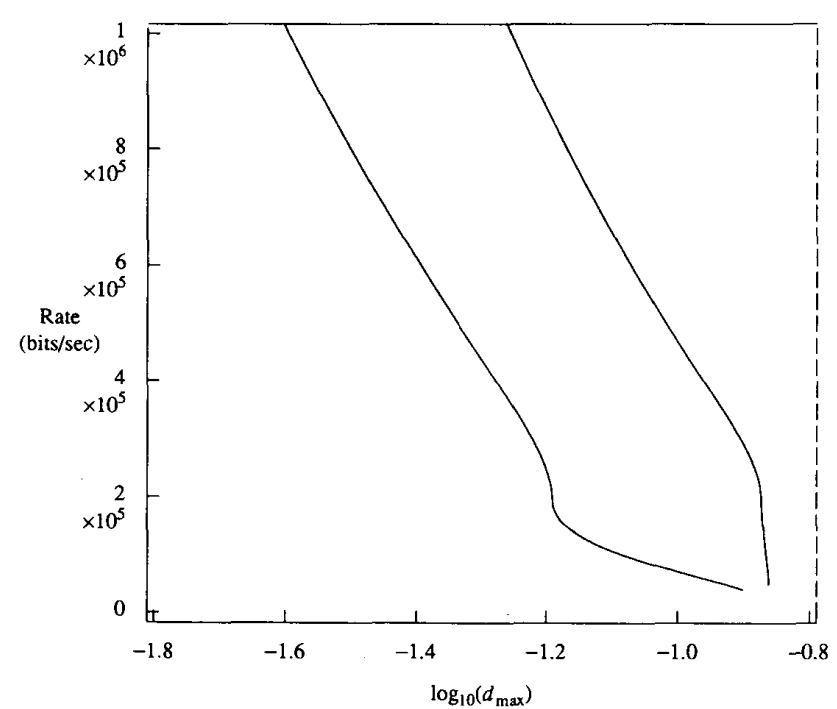

(c)

Fig. 5. (a) Subscriber loop impulse response. (b) Upper and lower bounds on $d_{\max }$ for impulse response in (a). (c) Upper and lower bounds on MCT for impulse response in (a). 
The impulse response for example 2 is shown in Fig. 4(a), and was computed from a transmission line model of 12 kilofeet of 24 gauge twisted-pair cable. This approximates a typical telephone subscriber loop impulse response without bridge taps. The impulse response is not oscillatory, and as shown in Fig. 4(b), the spread function and corresponding lower bound are monotonic. The impulse response for example 3 is shown in Fig. 5(a) and was computed from a transmission line model of a subscriber loop with bridge taps. The secondary peak in the impulse response is a reflection from a bridge tap.

For small receiver discriminations $d$, the upper and lower bounds on MCT obtained from Figs. 4(c) and 5(c) are within a factor of two to four. For the subscriber loop impulse response shown in Fig. 4(a), it appears that a receiver discrimination of $d=25$ mvolts allows a rate between approximately 800 and $1600 \mathrm{kbps}$. For the impulse response shown in Fig. 5(a), a rate of 1 Mbps can be achieved with a receiver discrimination between approximately 30 and $60 \mathrm{mV}$.

\section{Discussion}

The preceding numerical results indicate that the difference between the upper and lower bounds on MCT is greater for oscillatory types of signals than for "baseband" signals. This is due to the fact that for the oscillatory impulse response shown in Fig. 3(a), the derivative of the spread function near the origin increases with the frequency of oscillation. This is a direct consequence of the definition of spread, which is essentially the maximum amount the output amplitude can change in a given time in response to an amplitude limited input.

To be more specific, consider a channel strictly bandlimited to $[\omega-W / 2, \omega+W / 2]$, and any fixed $d<$ $2 \int_{0}^{\infty}|h(t)| d t$. It can be shown that the MCT and, of course, the lower bound, are bounded for all center frequencies $\omega$. However, the corresponding upper bound on MCT tends to infinity with $\omega$. Note that for this channel it is likely that the MCT depends on $\omega$. In contrast, this is not true of the Shannon capacity for a power-limited additive Gaussian noise channel, and also $\epsilon$-capacity, which assumes an $L_{2}$ constraint at the transmitter and the $L_{2}$ criterion to distinguish channel outputs [3].

We conclude by mentioning two alternative approaches to obtaining bounds on the MCT. The first approach is based on volume arguments, and is analogous to the technique used by Root to obtain upper and lower bounds on $\epsilon$-capacity [3]. In discrete-time the set of channel outputs in response to amplitude-limited inputs on a finite time interval is a parallelopiped in Euclidean space. Bounds on the MCT can be obtained by bounding the number of cubes, of length $d$ on a side, that can be packed into this parallelopiped. The resulting upper bound is likely to be looser than the spread bound for impulse responses that are "close" to $\mathrm{e}^{-t}$; however, it is likely to be tighter than the spread bound for oscillatory types of impulse responses.
Finally, one can, in principle, obtain an upper bound on the MCT for a channel with impulse response $h(t)$ by considering a channel with impulse response $h(t)$ followed by an additive noise source $n(t)$, where $|n(t)|<d / 2$. The Shannon capacity of this channel with amplitude constrained inputs must be at least as large as MCT $(d)$ evaluated for $h(t)$. This is easily shown by applying the set of inputs that achieves the $\operatorname{MCT}(d)$ to the preceding channel with noise. The receiver selects the message $j$ corresponding to the channel output $h * u_{j}$, which is closest in $L_{x}$ norm to the received signal. MCT $(d)$ is therefore an achievable rate in the Shannon sense. This is true for any random process $n(t)$ such that $|n(t)|<d / 2$ for all $t$. The analogous approach in the context of $\epsilon$-capacity is considered in [9]. Of course, evaluating the Shannon capacity of the preceding channel with specific noise statistics and amplitude constrained inputs is likely to be very difficult.

\section{APPENDIX \\ PROOF OF THEOREM 2}

The proof consists of two parts. In the first, we consider a class of impulse response functions $h(t)$ for which $\sigma^{+}$and $\sigma^{-}$ have certain properties, which are listed next. For any impulse response in this class, we first prove that

$$
T_{\min }(N, d) \geq n \tilde{\tau}
$$

where $\tilde{\tau}$ is defined by (3.7). The second part of the proof then shows how the proof in part one can be extended to apply to an arbitrary impulse response $h(t)$ by using the functions $\bar{S}^{+}$and $\bar{S}^{-}$defined by (3.4) instead of $\sigma^{+}$and $\sigma^{-}$. Following the proof of Theorem 2 we subsequently prove conditions for which $\tilde{\tau}=\tau^{*}$, where $\tau^{*}$ is defined by (3.6)

Let $\boldsymbol{H}$ denote a set of impulse response functions that have the following properties, i.e., $h(\cdot) \in \boldsymbol{H}$ if and only if

1) $\sigma^{+}$and $\sigma^{-}$are continuous functions of $t$ and $\alpha$;

2) $\sigma^{+}(t, \alpha)$ is a nondecreasing function of $t$ for fixed $\alpha$ (Theorem 3 then implies that $\sigma^{-}(t, \alpha)$ is a nonincreasing function of $t$;

3) $\sigma^{+}(t, \alpha)$ is a nondecreasing function of $\alpha$ for fixed $t$ (Theorem 3 then implies that $\sigma^{-}(t, \alpha)$ is a nondecreasing function of $\alpha$ ); and

4) $0 \leq \sigma^{+}(t, \alpha)-\sigma^{+}\left[t-t_{0}, \sigma^{-}\left(t_{0}, \alpha\right)\right] \leq \sigma\left(t_{0}, \alpha\right)$ for any $\alpha \in$ $\left[-\|h\|,\|h\|_{1}\right]$ and $t \geq t_{0} \geq 0$.

The set $\boldsymbol{H}$ is not empty. In particular, property 1$)$ is satisfied if $h(\cdot)$ is bounded and piecewise continuous, and properties 2)-4) are satisfied for any positive nonincreasing impulse response (see [8] and Theorem 8).

We now prove (A.1) given that $h \in \boldsymbol{H}$. Let $y_{1}, \cdots, y_{N}$ be a set of outputs corresponding to an optimal set of inputs, i.e., the outputs are distinguished in the minimum time $T_{\min } \equiv T_{\min }(N, d)$. Consider the set of outputs for $\tau \leq t \leq T_{\min }$, where $\tau$ is any fixed time such that $0 \leq \tau<T_{\min }$. We will say that a set of outputs $A$ has not been distinguished at time $\tau$ if for any two outputs $y_{i} \in A$ and $y_{j} \in A,\left|y_{i}(t)-y_{j}(t)\right|<d$ for $t \leq \tau$. Define the sampling time for distinct outputs $y_{i}$ and $y_{j}$ as

$$
t_{i j}=\min _{t}\left\{t|| y_{i}(t)-y_{j}(t) \mid=d\right\} .
$$


Let $A(\tau)$ denote a set of outputs that has not been distinguished at time $\tau$, and let $t_{i}=t_{i}$, be the first sampling time for $y_{i}$ which is greater than $\tau$, where $y_{j} \in A(\tau)$, i.e.,

$$
t_{i}=\min _{j}\left\{t_{i j} \mid t_{i j}>\tau, y_{j} \in A(\tau)\right\} .
$$

The number of elements in the set $A(\tau)$ will be denoted as $|\boldsymbol{A}(\tau)|$.

Given some $\alpha_{0} \in\left[-\|h\|_{1},\|h\|_{1}\right]$ define the following subset of outputs,

$$
Y\left(\tau, \alpha_{0}\right)=\left\{y_{i} \mid \sigma^{-}\left(t_{i}-\tau, \alpha_{0}\right) \leq y_{i}\left(t_{i}\right) \leq \sigma^{+}\left(t_{i}-\tau, \alpha_{0}\right)\right\} .
$$

Given any $y_{i} \in Y\left(\tau, \alpha_{0}\right)$, we can replace it by the following function $\bar{y}_{i}$ for $\tau \leq t \leq T_{\min }$ :

$$
\tilde{y}_{i}(\tau)=\alpha_{0}, \quad \tilde{y}_{i}(t)=y_{i}(t), \quad t \geq t_{i} .
$$

Suppose $y_{i}$ is being compared with $y_{j}$ at the sampling time $t_{i}$. If $y_{i}\left(t_{i}\right) \geq y_{j}\left(t_{i}\right)$,

$$
\begin{aligned}
\tilde{y}_{i}(t) & =\left\{\begin{array}{ll}
\sigma^{+}\left(t-\tau, \alpha_{0}\right), & \tau \leq t<t_{i}^{\prime} \\
\sigma^{-}\left[t-t_{i}^{\prime}, \sigma^{+}\left(t_{i}^{\prime}-\tau, \alpha_{0}\right)\right], & t_{i}^{\prime} \leq t<t_{i}
\end{array} .\right. \\
\text { If } y_{i}\left(t_{i}\right) & <y_{j}\left(t_{i}\right), \\
\tilde{y}_{i}(t) & = \begin{cases}\sigma^{-}\left(t-\tau, \alpha_{0}\right), & \tau \leq t<t_{i}^{\prime} \\
\sigma^{+}\left[t-t_{i}^{\prime}, \sigma^{-}\left(t_{i}^{\prime}-\tau, \alpha_{0}\right)\right], & t_{i}^{\prime} \leq t<t_{i}\end{cases}
\end{aligned}
$$

where $t_{i}^{\prime}$ is selected so that $\tilde{y}_{i}\left(t_{i}\right)=y_{i}\left(t_{i}\right)$. The definition of $\boldsymbol{Y}\left(\tau, \alpha_{0}\right)$ and property 1$)$ ensures that such a $t_{i}^{\prime}$ exists. We will refer to this substitution of $\tilde{y}_{i}$ for $y_{i}$ as the left pinch substitution.

Lemma A.1: Given any subset of outputs, $\boldsymbol{A}(\tau)$, ihat has not been distinguished at time $\tau$, and such that $|A(\tau)|=M$ and $Y\left(\tau, \alpha_{0}\right) \supset A(\tau)$ for some $\alpha_{0}$, then $T_{\min }-\tau \geq n \tilde{\tau}$, where $2^{n-1}<$ $M \leq 2^{n}$.

Proof: The proof is by induction on the size of the set $A(\tau)$. Suppose that $|\boldsymbol{A}(\tau)|=2$. Denote the elements of $\boldsymbol{A}(\tau)$ as $y_{i}$ and $y_{j}$, and assume that $y_{i}\left(t_{i}\right)>y_{j}\left(t_{i}\right)$, where $t_{i}$ is defined by (A.3). Since $y_{i}$ and $y_{j}$ are elements of $Y\left(\tau, \alpha_{0}\right)$, we can perform the left pinch substitution giving $\tilde{y}_{i}$ and $\tilde{y}_{j}$. Let $t_{\min }^{\prime}=\min \left(t_{i}^{\prime}, t_{j}^{\prime}\right)$ and $t_{\max }^{\prime}=\max \left(t_{i}^{\prime}, t_{j}^{\prime}\right)$ where $t_{i}^{\prime}$ is defined in (A.6). Divide the interval $\left[\tau, t_{i}\right]$ into the subintervals $I_{1}=\left[\tau, t_{\text {min }}^{\prime}\right], I_{2}=\left[t_{\min }^{\prime}, t_{\text {max }}^{\prime}\right]$, and $I_{3}=\left[t_{\max }^{\prime}, t_{i}\right]$. Since $h(\cdot) \in \boldsymbol{H}$, property 2$)$ implies that $\tilde{y}_{i}(t)-\bar{y}_{j}(t)$ is a nonincreasing function for $t \in I_{3}$, so that $\tilde{y}_{i}\left(t_{\max }^{\prime}\right)-$ $\tilde{y}_{j}\left(t_{\max }^{\prime}\right) \geq d$. Replacing $t$ by $t_{\max }^{\prime}$ and $t_{0}$ by $t_{\min }^{\prime}$ in property 4) implies that $\tilde{y}_{i}\left(t_{\min }^{\prime}\right)-\tilde{y}_{j}\left(t_{\min }^{\prime}\right) \geq \tilde{y}_{i}\left(t_{\max }^{\prime}\right)-\tilde{y}_{j}\left(t_{\max }^{\prime}\right) \geq d$. Consequently, there exists a $t^{*} \leq t_{\min }^{\prime}$ such that $\tilde{y}_{i}\left(t^{*}\right)-\bar{y}_{j}\left(t^{*}\right)=d$. (A.6) therefore implies that

$$
\begin{aligned}
& \tilde{y}_{i}\left(t^{*}\right)=\sigma^{+}\left(t^{*}-\tau, \alpha_{0}\right), \\
& \tilde{y}_{j}\left(t^{*}\right)=\sigma^{-}\left(t^{*}-\tau, \alpha_{0}\right),
\end{aligned}
$$

and

$$
\dot{y}_{i}\left(t^{*}\right)-\tilde{y}_{j}\left(t^{*}\right)=\sigma\left(t^{*}-\tau, \alpha_{0}\right)=d .
$$

Since $\sigma\left(t^{*}-\tau, \alpha_{0}\right)<\sup _{\alpha} \sigma\left(t^{*}-\tau, \alpha\right)$, it follows that $T_{\min }-\tau \geq$ $t^{*}-\tau \geq \tilde{\tau}$, which proves the lemma for the case $|\boldsymbol{A}(\tau)|=2$.

Assume now that $|A(\tau)|=N$, and that Lemma A.1 is true for $|A(\tau)|=[N / 2]$, where $N$ is a positive integer. Denote the elements of $A(\tau)$ as $y_{1}, \cdots, y_{N}$. Since the members of $A(\tau)$ are also in $Y\left(\tau, \alpha_{0}\right)$, we can again perform the left pinch substitution giving the functions $\bar{y}_{1}, \cdots, \bar{y}_{N}$. Observe that the argument preceding (A.7) again applies to each $\tilde{y}_{i}, i=1, \cdots, N$, so that $\tilde{y}_{i}\left(t^{*}\right)$ satisfies either (A.7a) or (A.7b) for each $i=1, \cdots, N$. Denote the set of outputs $y_{i}$ corresponding to all $\tilde{y}_{i}$ 's that satisfy (A.7a) as
$P_{1}$ and the set of $y_{i}$ corresponding to all $\tilde{y}_{i}$ 's that satisfy (A.7b) as $\boldsymbol{P}_{2}$, and observe that neither of these sets can be empty.

We now show that the set $\boldsymbol{P}_{1}\left(\boldsymbol{P}_{2}\right)$ satisfies the conditions of Lemma A.1 for $\tau=t^{*}$ and $\alpha_{0}=\tilde{y}_{i}\left(t^{*}\right)$ where $y_{i} \in \boldsymbol{P}_{1}\left(\boldsymbol{P}_{2}\right)$. In particular, we show that the set $\boldsymbol{P}_{1}$ has not been distinguished at time $t^{*}$, and that $y_{i} \in \boldsymbol{Y}\left[t^{*}, \bar{y}_{i}\left(t^{*}\right)\right]$. Let $t_{\min }=\min _{i} t_{i}$. Observe that $t^{*}$ is independent of any particular output $y_{i}$, and that $t^{*} \leq t_{\min }$. If $t^{*}<t_{\min }$, then the original set of outputs $A(\tau)=\boldsymbol{P}_{1}$ $\cup \boldsymbol{P}_{2}$ has not been distinguished at time $t^{*}$. If $t^{*}=t_{\min }$, then at time $t^{*}$ any member of $\boldsymbol{P}_{1}$ is separated by $d$ from any member of $\boldsymbol{P}_{2}$; however, each set of outputs $\boldsymbol{P}_{1}$ and $\boldsymbol{P}_{2}$ has not been distinguished. (Otherwise, there would exist some sampling time $t_{i}<t_{\text {min }}$. Consequently, there are at least $\max \left(\left|\boldsymbol{P}_{1}\right|,\left|\boldsymbol{P}_{2}\right|\right) \geq\lceil N / 2]$ outputs that have not been distinguished at $t=t^{*}$.

Suppose that $y_{i} \in \boldsymbol{P}_{1}$. The assumption $y_{i} \in \boldsymbol{Y}\left(\tau, \alpha_{0}\right)$ implies that

$$
\begin{aligned}
y_{i}\left(t_{i}\right) \leq & \sigma^{+}\left(t_{i}-\tau, \alpha_{0}\right) . \\
= & \sup _{\substack{u(s) \\
-\infty<s \leq t_{i}-\tau}}\left\{y\left(t_{i}-\tau\right)\left|y(0)=\alpha_{0},\right| u(s) \mid \leq 1\right\} .
\end{aligned}
$$

Assume that the supremum is achieved by the input $u^{*}(s)$, and let $y^{*}\left(t^{*}\right)$ denote the associated output value at time $t^{*}$. Observe that $y^{*}\left(t^{*}\right) \leq \sigma^{+}\left(t^{*}-\tau, \alpha_{0}\right)=\bar{y}_{i}\left(t^{*}\right)$. Consequently,

$$
\begin{aligned}
\sigma^{+}\left(t_{i}-\tau, \alpha_{0}\right) & \\
\leq & \sup _{\substack{u(s) \\
-\infty<s \leq t_{i}-t^{*}}}\left\{y\left(t_{i}-t^{*}\right)\left|y(0)=y^{*}\left(t^{*}\right),\right| u(s) \mid \leq 1\right\} \\
= & \sigma^{+}\left[t_{i}-t^{*}, y^{*}\left(t^{*}\right)\right] \leq \sigma^{+}\left[t_{i}-t^{*}, \tilde{y}_{i}\left(t^{*}\right)\right], \quad \text { (A.10) }
\end{aligned}
$$

where the last inequality follows from property 3 ). Also, since $t_{i}-t^{*} \geq t_{i}-t_{i}^{\prime}$ where $t_{i}^{\prime}$ is defined by (A.6), and $\bar{y}_{i}\left(t^{*}\right) \leq \tilde{y}_{i}\left(t_{i}^{\prime}\right)$ (follows from property 2$)$ ), properties 2 ) and 3 ) imply that

$$
y_{i}\left(t_{i}\right)=\sigma^{-}\left[t_{i}-t_{i}^{\prime}, \tilde{y}_{i}\left(t_{i}^{\prime}\right)\right] \geq \sigma^{-}\left[t_{i}-t^{*}, \tilde{y}_{i}\left(t^{*}\right)\right] .
$$

(A.9)-(A.11) imply that $y_{i} \in \boldsymbol{Y}\left[t^{*}, \tilde{y}_{i}\left(t^{*}\right)\right]$. It is easily verified that this remains true if $y_{i} \in \boldsymbol{P}_{2}$. Consequently, the induction assumption implies that $T_{\min }-\tau \geq\left(t^{*}-\tau\right)+n \tilde{\tau} \geq(n+1) \tilde{\tau}$ where $2^{n-1}<[N / 2] \leq 2^{n}$, or equivalently, $2^{n}<N \leq 2^{n+1}$.

To complete the first part of the proof we need only verify that any set of $N$ outputs that are distinguished in time $T_{\min }(N, d)$ must satisfy the conditions of Lemma A.1 at $t=0$. Since $y_{i}(0)=0$ for each $i$, the set of outputs $y_{i}, i=1, \cdots, N$, has not been distinguished at $t=0$. Finally, the definition of $\sigma^{ \pm}$ implies that $y_{i}(t) \in Y(0,0)$ for each $i=1, \cdots, N$. This completes the proof that $\mathrm{MCT} \leq 1 / \tilde{\tau}$ for $h \in \boldsymbol{H}$.

We now complete the proof of Theorem 2 by showing that the proof of Lemma A.1 can be extended to arbitrary $h(t)$ by substituting $\bar{S}^{+}$and $\bar{S}^{-}$, defined by (3.3) and (3.4), for $\sigma^{+}$and $\sigma^{-}$, respectively. Of course, $\bar{\tau}$ in the statement of Lemma A.1 must be replaced by $\tau^{*}$ defined by (3.6). It is easily verified that the proof of Lemma A.1 remains valid if $\bar{S}^{ \pm}$satisfies the following properties.

1) $\sigma^{+}(t, \alpha) \leq \bar{S}^{+}(t, \alpha)$ and $\sigma^{-}(t, \alpha) \geq \bar{S}^{-}(t, \alpha), \quad$ for all $t \geq 0$ and $\alpha \in\left[-\|h\|_{1},\|h\|_{1}\right]$.

2) $\bar{S}^{ \pm}$satisfies properties 1)-4) satisfied by $\sigma^{ \pm}$when $h \in \boldsymbol{H}$.

3) $\bar{S}^{+}(t, \alpha) \leq \bar{S}^{+}\left[t-t_{0}, \bar{S}^{+}\left(t_{0}, \alpha\right)\right]$.

(A.12)

This guarantees that (A.10) remains true when $\sigma^{+}$is replaced by $\bar{S}^{+}$, for $t \geq t_{0}>0$. In what follows we still assume that $h(\cdot)$ is bounded and piecewise continuous so that $\bar{S}^{ \pm}$is continuous.

We now prove the preceding statements, which will establish Theorem 2. Property 1) follows directly from the definition of 
$\bar{S}^{ \pm}$, and (A.12) follows directly from (A.10) and definitions (3.3) and (3.4). The next lemma establishes property 2 ).

Lemma A.2: $\bar{S}^{+}(t, \alpha)$ is a nondecreasing function of $t$. for fixed $\alpha$, and a nondecreasing function of $\alpha$ for fixed $t$. Also,

1) $\bar{S}^{+}(t, \alpha)=-\bar{S}^{-}(t,-\alpha)$

2) $0 \leq \bar{S}^{+}(t, \alpha)-\bar{S}^{+}\left[t-t_{0}, \bar{S}^{-}\left(t_{0}, \alpha\right)\right] \leq \bar{S}\left(t_{0}, \alpha\right), \quad$ for any $t \geq t_{0}>0$.

Proof: The definition (3.3) directly implies that $S^{+}(t, \alpha)$ is a nondecreasing function of $t$ for fixed $\alpha$ and a nondecreasing function of $\alpha$ for fixed $t$. Consequently, (3.4) implies that $\bar{S}^{+}(t, \alpha)$ is a nondecreasing function of $t$ for fixed $\alpha$. Assume that $\bar{S}^{+}\left(t, \alpha_{1}\right)<\bar{S}^{+}\left(t, \alpha_{2}\right)$ for $\alpha_{1}>\alpha_{2}$. Definition (3.4) implies that there exists an $\alpha_{2}^{\prime} \geq \alpha_{2}$ such that

$$
\alpha_{1}+\sup _{\alpha_{1}^{\prime} \geq \alpha_{1}}\left(S^{+}\left(t, \alpha_{1}^{\prime}\right)-\alpha_{1}^{\prime}\right)<\alpha_{2}+\left(S^{+}\left(t, \alpha_{2}^{\prime}\right)-\alpha_{2}^{\prime}\right) .
$$

If $\alpha_{2}^{\prime} \geq \alpha_{1}$, then taking $\alpha_{1}^{\prime}=\alpha_{2}^{\prime}$ leads to a contradiction. Consequently, $\alpha_{2} \leq \alpha_{2}^{\prime}<\alpha_{1}$. But this implies that

$$
\begin{aligned}
S^{+}\left(t, \alpha_{1}\right) & \leq \bar{S}^{+}\left(t, \alpha_{1}\right)<\bar{S}^{+}\left(t, \alpha_{2}\right) \\
& =\alpha_{2}+S^{+}\left(t, \alpha_{2}^{\prime}\right)-\alpha_{2}^{\prime} \leq S^{+}\left(t, \alpha_{2}^{\prime}\right),
\end{aligned}
$$

which is a contradiction since $\alpha_{2}^{\prime}<\alpha_{1}$ and $S^{+}(t, \alpha)$ is a nondecreasing function of $\alpha$. This establishes that $\bar{S}^{+}(t, \alpha)$ is a nondecreasing function of $\alpha$.

Statement 1) follows directly from Theorem 3 and (3.3), (3.4). To prove 2), observe that the definition (3.4) implies that $\bar{S}^{+}\left(t-t_{0}, \alpha_{1}\right)-\bar{S}^{+}\left(t-t_{0}, \alpha_{2}\right)$ is a nonincreasing function of $t$ for $\alpha_{1}>\alpha_{2}$ and $t \geq t_{0} \geq 0$. Taking $\alpha_{1}=\bar{S}^{+}\left(t_{0}, \alpha\right)$ and $\alpha_{2}=$ $\bar{S}^{-}\left(t_{0}, \alpha\right)$, (A.12) implies that

$$
\begin{aligned}
\alpha_{1}-\alpha_{2} & =\bar{S}\left(t_{0}, \alpha\right) \geq \bar{S}^{+}\left(t-t_{0}, \alpha_{1}\right)-\bar{S}^{+}\left(t-t_{0}, \alpha_{2}\right) \\
& \geq \bar{S}^{+}(t, \alpha)-\bar{S}^{+}\left(t-t_{0}, \alpha_{2}\right) \geq 0
\end{aligned}
$$

for $t \geq t_{0}$, where the last inequality follows from the facts that $\alpha_{2}=\bar{S}^{-}\left(t_{0}, \alpha\right) \leq \alpha$ and $\bar{S}^{+}(t, \alpha)$ is a nondecreasing function of $t$ and $\alpha$.

Remark: The proof of Lemma A.1 can be modified slightly to improve the lower bound on $T_{\min }$ given by Theorem 2. Define a set of bifurcating functions $f_{1}, \cdots, f_{N}$ as follows.

1) $f_{i}(0)=\alpha$ for each $i$, where $-\|h\|_{1} \leq \alpha \leq\|h\|_{1}$. Define the separation time between $f_{1}$ and $f_{j}$ as

$$
\tau_{1 j}=\min \left\{t|| f_{1}(t)-f_{j}(t) \mid=d\right\},
$$

and assume that $\tau_{1, m_{1}}<\tau_{1, m_{2}}<\cdots<t_{1, m_{N-1}}$, are the successive separation times for $f_{1}$. (Separation time is used instead of sampling time to emphasize the distinction between $y_{j}$ and $f_{j}$.)

2) Either $f_{1}(t)=\bar{S}^{+}\left[t, f_{1}\left(t_{1, m_{j}}\right)\right]$ or $f_{1}(t)=\bar{S}^{-}\left[t, f_{1}\left(t_{1, m_{j}}\right)\right]$ for $t_{1, m_{j}}<t<t_{1, m_{l+1}}, j=1, \cdots, N-2$.

$f_{2}, \cdots, f_{N}$ are defined similarly.

The number of functions that increase (decrease) at each separation time has not been specified. This is determined by some rule $R$. According to a particular rule, $\hat{R}$, if $m$ functions are the same for $0 \leq t \leq \bar{t}$, where $\bar{t}$ is a separation time, then $\lfloor m / 2\rfloor$ functions are equal to $\bar{S}^{+}$at $t=\tilde{t}^{+}$.

Let $T_{h i f}(N, d, \alpha ; R)$ be the time it takes to distinguish $N$ functions $f_{1}, \cdots, f_{N}$ given by the previous construction, assuming that the functions bifurcate according to rule $R$. Clearly, for any rule $R$,

$$
T_{b i f}\left(2^{m}, d, \alpha ; R\right) \geq m \tau^{*},
$$

where $\tau^{*}$ is defined by (3.6). It is easily verified that the preceding proof can be slightly modificd to show that

$$
T_{\min }(N, d) \geq \min _{R} T_{h i f}(N, d, 0 ; R) .
$$

This bound is tighter than the upper bound given by Theorem 2; however, it is more difficult to compute for arbitrary impulse response $h$.

We now derive conditions on the impulse response $h(\cdot)$ that imply $\tau^{*}=\tilde{\tau}$.

Theorem 7: For any impulse response $h$ and any $t \geq 0$, $\sup _{\alpha} S(t, \alpha)=S(t, 0)=\sup _{0 \leq t^{\prime} \leq t} \sigma\left(t^{\prime}, 0\right)$.

Note that Theorem 7 and (3.4) imply that $\sup _{\alpha} \bar{S}(t, \alpha)=\bar{S}(t, 0)$. Theorem 7, the definition of $\tau^{*}$, and the fact that $S(t, \alpha)$ is a nondecreasing function of $t$ imply that if $\bar{S}\left(\tau^{*}, 0\right)=S\left(\tau^{*}, 0\right)$, then $\bar{S}\left(\tau^{*}, 0\right)=\sigma\left(\tau^{*}, 0\right)$, which implies that $\tau^{*}=\tilde{\tau}$. To prove Theorem 7 the following lemma is needed.

Lemma A.3: For any impulse response $h$ and fixed time $t$

$$
\sup _{\alpha} \sigma^{+}(t, \alpha)=\sigma^{+}\left(t, \alpha_{0}\right)=\|h\|_{1},
$$

where $\alpha_{0}(t)=\int_{-\infty}^{0} \operatorname{sgn}[h(t-s)] h(-s) d s$.

Proof: Choose the input $u(t)=\operatorname{sgn}[h(t-s)]$ and note that $\sigma^{+}(t, \alpha) \leq\|h\|_{1}$ for any $t$ and $\alpha$.

Proof of Theorem 7: Theorem 3 and the definition of $S^{ \pm}$, (3.3), imply that $S(t, \alpha)$ is a concave and even function of $\alpha$, which implies the left equality. To prove the right equality it is sufficient to show that

$$
S^{+}(t, 0)=\sup _{\substack{0 \leq t^{\prime} \leq t \\-\|h\|_{1} \leq \alpha^{\prime} \leq 0}} \sigma^{+}\left(t^{\prime}, \alpha^{\prime}\right)=\sup _{0 \leq t^{\prime} \leq t} \sigma^{+}\left(t^{\prime}, 0\right) .
$$

Theorem 3 and Lemma A.2 will then imply that $S^{-}(t, 0)=$ $\sup _{0 \leq t^{\prime} \leq t} \sigma^{-}\left(t^{\prime}, 0\right)$. Suppose (A.20) is false. Then there exists a time $\bar{t} \leq t$ and an $\alpha^{\prime}<0$ such that

$$
\sigma^{+}\left(\bar{t}, \alpha^{\prime}\right)>\sup _{0 \leq t^{\prime} \leq t} \sigma^{+}\left(t^{\prime}, 0\right) \geq \sigma^{+}(\tilde{t}, 0) .
$$

Since $\sigma^{+}$is a concave function of $\alpha$, it has a unique maximum at $\alpha_{0}$ defined in Lemma A.3, and is an increasing function of $\alpha$ for $-\|h\|_{1} \leq \alpha \leq \alpha_{0}$. Therefore (A.21) implies that $\alpha_{0}(\tilde{t}) \leq 0$. It is casily shown from the definition that $\alpha_{0}(t)$ is a continuous function of $t$ for bounded and piecewise continuous $h(\cdot)$. Furthermore, $\alpha_{0}(0)=\|h\|_{1}>0$. Consequently, there exists some time $t_{0} \leq \hat{t}$ for which $\alpha_{0}\left(t_{0}\right)=0$, which implies that $\sup _{0 \leq t^{\prime} \leq t} \sigma^{+}\left(t^{\prime}, 0\right)=\sigma^{+}\left(t_{0}, 0\right)=\|h\|_{1}$, which contradicts (A.21).

Theorem 8: Suppose that $\sigma(t, 0)=S(t, 0)$. Then $\bar{S}(t, 0)=$ $\sigma(t, 0)$ if and only if

$$
\int_{\boldsymbol{B}(t)}|h(-s)| d s \leq \int_{\bar{B}(t)}|h(-s)| d s .
$$

where $\boldsymbol{B}(t)$ and $\overline{\boldsymbol{B}}(t)$ are defined by (3.9).

Theorems 7 and 8 imply that if (A.22) is true for $t=\tau^{*}$, and if $\sigma\left(\tau^{*}, 0\right)=\sup _{0 \leq t^{\prime} \leq \tau^{*}} \sigma\left(t^{\prime}, 0\right)$, then $\tau^{*}=\tilde{\tau}$

Proof: Theorem 3 implies that it is sufficient to show that (A.22) is true if and only if $\bar{S}^{+}(t, 0)=\sigma^{+}(t, 0)$. Since $\sigma^{+}(t, 0)=$ $S^{+}(t, 0), \bar{S}^{+}(t, 0)=\sigma^{+}(t, 0)$ if $f(t, \alpha)=\sigma^{+}(t, \alpha)-\alpha$ is monotonically decreasing in $\alpha$ for $0 \leq \alpha \leq\|h\|_{1}$. Since $\sigma^{+}$is a concave function of $\alpha, \partial f / \partial \alpha$ is a decreasing function of $\alpha$ for $\alpha \geq 0$. 
Therefore $\sigma^{+}(t, 0)=\bar{S}^{+}(t, 0)$ if and only if

$$
\left.\frac{\partial \sigma^{+}(t, \alpha)}{\partial \alpha}\right|_{\alpha=0} \leq 1
$$

It is shown in [8] that $\partial \sigma^{+}(t, \alpha) / \partial \alpha=\lambda(t, \alpha)$, where

$$
\begin{aligned}
& \int_{h(t-s) / h(-s)>\lambda, s \leq 0}|h(-s)| d s \\
&-\int_{h(t-s) / h(-s)<\lambda, s \leq 0}|h(-s)| d s=\alpha,
\end{aligned}
$$

and that $\lambda$ is a monotonically decreasing function of $\alpha$. Consequently, $\bar{S}(t, 0)=\sigma(t, 0)$ if and only if $\lambda(t, 0) \leq 1$, which combined with (A.24) implies (A.22).

\section{REFERENCES}

[1] R. V. L. Hartley, "Transmission of information," Bell Syst. Tech. J., vol. 7 , no. 3, pp. $535-563$, July 1928.

[2] J. M. Wozencraft and I. M. Jacobs, Principles of Communication Engineering. New York: John Wiley, pp. 2ff, 1965.

[3] W. L. Root, "Estimates of $\epsilon$-capacity for certain linear communication channels," IEEE Trans. Inform. Theory, vol. IT-14, pp. 361-369, May 1968

[4] M. L. Honig, S. Boyd, B. Gopinath, and E. Rantapaa, "On optimum signal sets for digital communications with finite precision and amplitude constraints," submitted to IEEE Trans. Communications.

[5] P. Bloomfield and W. L. Steiger, Least Absolute Deviations. Boston: Birkhauser, 1983.

[6] C. Witzgall, "On one-row linear programs," in Extremal Methods and Systems Analysis, A. V. Fiacco and K. O. Kortanek, Eds. New York: Springer-Verlag, pp. 384-414, 1980.

[7] M. L. Honig and B. Gopinath, "Estimates of maximum throughput for simulated local loops," submitted to the 1988 Globecom Conf., Hollywood, FL.

[8] A. Friedman and M. L. Honig, "On the spread of continuous-time linear systems," SIAMJ. Math. Anal., to appear.

[9] L. J. Forys and P. P. Varaiya, "The $\epsilon$-capacity of classes of unknown channels," Inform. Contr., vol. 14, pp. 376-406, 1969. 